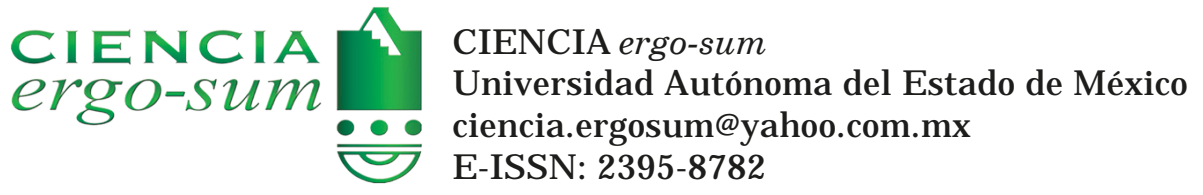

\title{
Estudio sobre competencias digitales en programas de formación virtual y a distancia
}

Silva Monsalve, Alexandra María; Martínez Morales, Edgar Mauricio; Ortega Ferreira, Sandra Constanza;
Mejía Corredor, Carolina; Maldonado Currea, Adriana
Estudio sobre competencias digitales en programas de formación virtual y a distancia
CIE NCIA ergo-sum, vol. 28, núm. 3, noviembre 2021-febrero 2022| e132
Universidad Autónoma del Estado de México, México
Esta obra está bajo una Licencia Creative Commons Atribución-NoComercial-SinDerivar 4.0 Internacional.

Silva Monsalve, A. M., Martínez Morales, E. M., Ortega Ferreira, S. C., Mejía Corredor, C. y Maldonado Currea, A. (2021). Estudio sobre competencias digitales en programas de formación virtual y a distancia. $\mathrm{CIE} \mathrm{NCIA} \mathrm{ergo-sum,}$ 28(3). https://doi.org/10.30878/ces.v28n3a4 


\title{
Estudio sobre competencias digitales en programas de formación virtual y a distancia ${ }^{[1]}$
}

\section{Study on digital competences in virtual and distance training programs}

\author{
Alexandra Maria Silva Monsalve \\ Universidad Santo Tomás, Colombia \\ alexandrasilva@ustadistancia.edu.co \\ (D) http://orcid.org/0000-0001-7554-0237 \\ Edgar Mauricio Martinez Morales \\ Universidad Santo Tomás, Colombia \\ edgar.martinez@ustadistancia.edu.co \\ (D) http://orcid.org/0000-0003-1848-8107 \\ Sandra Constanza Ortega Ferreira \\ Escuela de Administración de Negocios, Colombia \\ scortega@universidadean.edu.co \\ (D) http://orcid.org/0000-0002-8662-6201 \\ Carolina Mejía Corredor \\ Escuela de Administración de Negocios, Colombia \\ cmejiaco@universidadean.edu.co \\ (D) http://orcid.org/0000-0002-3560-5443 \\ Adriana Maldonado Currea \\ Escuela de Administración de Negocios, Colombia \\ amaldonadoc@universidadean.edu.co \\ (D) http://orcid.org/0000-0002-8059-0148
}

\section{Resumen.}

Se examinan las fortalezas y las debilidades en el manejo tecnológico para programas en las modalidades virtual y a distancia, ya que las competencias digitales se han convertido en una habilidad necesaria para la educación en el siglo XXI. Metodológicamente, se lleva a cabo una investigación de tipo mixto y una revisión de la literatura para establecer un referente conceptual sobre competencias digitales. Asimismo, se identifica un instrumento para su validación, el cual se aplicó a estudiantes de dos universidades. Los resultados permiten establecer un referente conceptual situado en seis dimensiones y evidencian las categorías resultantes y su incidencia con el proceso de enseñanza y aprendizaje.

PALABras ClaVE: competencias digitales, educación abierta, educación tecnológica, destrezas básicas, formación en línea.

\section{Abstract}

The strengths and weaknesses in technological management for programs in virtual and remote modalities are examined, because the digital skills have become a necessary skill for education in the 21 st century. Methodologically, a mixed research and a literature review are carried out to establish a conceptual reference on digital skills. An instrument was identified for its validation, which was applied to students from two universities. The results allowed establishing a conceptual reference located in six dimensions and evidencing the resulting categories and their incidence with the teaching and learning process.

KEYWORDS: Digital competences, open education, technological education, basic skills, online training. 


\section{INTRODUCCIÓN}

El manejo de las competencias digitales (CD) para programas de formación en línea se ha convertido en una habilidad indispensable tanto para estudiantes como para docentes. No poseer las destrezas suficientes en el manejo de las tecnologías de la información y la comunicación (TIC) puede llevar al bajo rendimiento académico en este tipo de metodologías. El artículo indaga sobre el nivel de CD que tienen los estudiantes y docentes de las universidades para el caso específico de la Universidad Santo Tomás y la Escuela de Administración de Negocios (en adelante se denominarán USTA y EAN respectivamente). En este orden de ideas, primero se presenta la conceptualización desde el ámbito de las TIC, le sigue un recorrido sobre las CD en el contexto europeo y latinoamericano y para finalizar se asume el concepto para esta investigación seleccionando el marco de las CD que se evaluarán a los estudiantes y docentes que permitan establecer aspectos orientadores de formación en línea y se constituyan en un referente para otros programas.

\section{Las TIC y las COMPETENCIAS Digitales}

Las tecnologías de la información y la comunicación (TIC) se han constituido como un papel mediador para la enseñanza y el aprendizaje. Precisamente, el auge tecnológico en el contexto educativo ha posibilitado el surgimiento de nuevas competencias para su manejo. Muñoz Rojas (2016: 8) plantea:

Lo pedagógico se puede transformar a través de la mediación de las tecnologías agregando nuevas posibilidades y limitaciones al aprendizaje. En ese sentido, las tecnologías que participan en un proceso educativo pueden considerarse como sistemas de actuación (acción externa), pero también como fuente para la generación de nuevos modelos cognitivos o marcos de pensamiento (representación interna).

De esta manera, los programas en línea se han venido fortaleciendo junto con las CD y se han erigido en papel mediador para la enseñanza y el aprendizaje; sin estas competencias, el estudiante o docente no puede tener acceso pleno a los materiales o a presentar actividades que son requeridas y que se manejan en las plataformas virtuales. Por esta razón, los procesos de enseñanza-aprendizaje deben encontrar armonía con las mediaciones tecnológicas (Muñoz Rojas, 2016).

El concepto de CD ha ido evolucionando, pues en un inicio se limitaba a ser una habilidad funcional en el entorno del computador y la internet. Se proponía comprenderla como alfabetización digital en los siguientes términos:

Es la conciencia, la actitud y la capacidad de las personas para utilizar adecuadamente las herramientas digitales para identificar, acceder, administrar, integrar, evaluar, analizar y sintetizar los recursos digitales, construir nuevos conocimientos, expresarse a través de los recursos multimedia y comunicarse con los demás en cualquier contexto específico de la vida (Esteve y Gisbert, 2013).

Sin embargo, resultó insuficiente entenderlas debido a que la dimensión operativa es sólo una parte de ella y no respondía a las implicaciones del desarrollo de las TIC en todas sus dimensiones, específicamente de la web 2.0 que permite a los usuarios interactuar y colaborar. El uso de las CD se extendió a fenómenos como la comunicación interplanetaria, la producción, circulación y validación del conocimiento y reformuló su concepto. Así es como:

La CD implica el uso crítico y seguro de las tecnologías de la sociedad de la información para el trabajo, el tiempo libre y la comunicación. Apoyándose en habilidades TIC básicas: uso de ordenadores para recuperar, evaluar, almacenar, producir, presentar e intercambiar información, y para comunicar y participar en redes de colaboración a través de Internet (INTEF, 2017). 
En 2006, el Consejo y el Parlamento Europeo propusieron un marco de referencia que identificaba y definía las competencias claves que necesitan los ciudadanos para su realización personal, inclusión social, ciudadanía activa y empleabilidad en la sociedad del conocimiento. Por consiguiente, se establecieron ocho competencias: a) comunicación en la lengua materna, $b$ ) comunicación en lenguas extranjeras, $c$ ) competencia matemática y competencias básicas en ciencia y tecnología, $d$ ) competencia digital, $e$ ) aprender a aprender, $f$ ) competencias sociales y cívicas, $g$ ) sentido de la iniciativa y espíritu de empresa, $h$ ) conciencia y expresión culturales (Figel, 2009). En esta formulación se puede ubicar el lugar de reconocimiento que comienzan a adquirir las CD en la formación integral de los ciudadanos.

\section{Competencias digitales en Europa y Latinoamérica}

Para la determinación de las competencias digitales, en Europa se creó en 2011 el proyecto DIGCOMP: A Framework for Developing and Understanding Digital Competence in Europe, donde se realizaron distintos informes, incluyendo un marco de referencia sobre la construcción de currículos y certificaciones. Este proyecto de formulación de competencias y descriptores estuvo pensado para la ciudadanía en general y presentó en 2013 las siguientes áreas: $a$ ) información, $b$ ) comunicación, $c$ ) creación de contenido digital, $d$ ) seguridad, e) resolución de problemas. A su vez, cada una de ellas se dividía en subáreas o subcompetencias. Una de las razones que se pretende al establecer estándares consiste en la necesidad de validar el aprendizaje previo de los estudiantes, pues "en la época de los videos tutoriales, el contenido abierto y las redes sociales, resulta fácil adquirir habilidades" (NMC Horizon, 2016).

Seguidamente, en la versión 2017 del Informe Horizon se destacan dos tendencias claves que aceleran la adopción de nuevas tecnologías en la educación. La primera es el diseño de aprendizaje híbrido (blended learning), que con el tiempo se le ha dado reconocimiento a las ventajas de la educación en línea, por lo que se ha extendido esta propuesta a la educación presencial; de alguna manera, es la tendencia que parece imponerse. La segunda es el rediseño de los espacios de aprendizaje que ha dado origen a unas nuevas formas de organizar la instalación de pantallas y distintos dispositivos que contribuyen a ejecutar actividades donde los estudiantes realizan experimentos mediante contenidos holográficos en tercera dimensión (3D) con ambientes reales, encuentros virtuales para interactuar de manera colaborativa y ubicua y construir proyectos favorables al desarrollo de la innovación y al pensamiento crítico. Asimismo, la versión 2018 propone tendencias en aspectos como tecnologías de aprendizaje adaptativo, inteligencia artificial, realidad mixta y robótica (New Media Consortium-EDUCAUSE, 2018).

En Latinoamérica, la indagación por las CD se acentúa en especial en la formación docente; adicionalmente, en su formulación ubican el contexto social y económico sin los cuales no podrían tener sentido. En Ecuador se estableció que en la formación en competencias debe confluir en educación "la requerida para impulsar el desarrollo humano del Ecuador en función de la riqueza cultural y biodiversidad, así como las competencias tecnológicas que deben adquirirse a lo largo de la formación para estar integrados en las dinámicas de la globalización" (Pérez et al., 2017: 311). Desde la política pública de Colombia se encuentra que frente a las CD no se adopta una postura mecánica al pretender formar a sus nuevas generaciones para el mundo globalizado, sino destacar que su desarrollo debe estar en la tensión crítica entre el mundo globalizado y las aspiraciones locales que tienen los pueblos.

Por otra parte, en Argentina, las competencias se inscriben en el Plan Nacional Integral de Educación Digital (PLANIED), donde se propone:

Entender las TIC como formas culturales, como espacios en los cuales no solo circula información, sino también las distintas dimensiones que posibilitan configurar la subjetividad y construir conocimiento. En el espacio simbólico de las TIC, convergen tanto el juego, la exploración, la creatividad y la fantasía como el pensamiento crítico, la información, la comunicación y la colaboración, debiendo entenderse estas categorías como un todo integrado (PLANIED, 2016). 
En Colombia el Ministerio de Educación Nacional elaboró un documento titulado Las competencias TIC para el desarrollo profesional docente. Allí se presentan las competencias tecnológicas como "la capacidad para seleccionar y utilizar de forma pertinente, responsable y eficiente una variedad de herramientas tecnológicas entendiendo los principios que las rigen, la forma de combinarlas y las licencias que las amparan" (Ministerio de Educación Nacional, 2013: 36)

Según lo expuesto, a las CD no se les considera aisladas, puesto que se vinculan a otras como la comunicativa, la investigativa, la de gestión y la pedagógica. En este sentido, en Colombia se encuentra una mirada que concuerda con la europea al señalar que "la alfabetización digital no es un listado de habilidades técnicas específicas, sino más bien el desarrollo de un pensamiento crítico y la reflexión en diversos contextos sociales y culturales" (Informe Horizon, 2016).

No obstante, existe una brecha digital significativa entre las regiones urbanas y rurales, entre los estratos socioeconómicos altos y bajos y entre los grupos étnicos y culturales que dificultan el acceso a las TIC y a su uso cotidiano. Así, el informe de la Unión Internacional de Telecomunicaciones (UIT) de 2017 menciona que la reducción de la brecha digital entre los países más y menos conectados sigue constituyendo un desafío. La brecha entre los países situados en los segmentos superior e inferior de esta clasificación aumentó hasta situarse en los 8.02 puntos (sobre 10) en 2017. Al igual que en años anteriores, existe una estrecha correlación entre los niveles de desarrollo económico y de las TIC, y los países menos adelantados PMA ocupan 37 de los 44 puestos del cuartil inferior (menos conectado) de la clasificación (International Telecommunications Union, 2017). Ahora bien, el informe presenta otros aspectos determinantes para seguir consolidando estrategias en materia de acercamiento de la brecha digital (tabla 1$)$.

TABLA 1

Tendencias emergentes en materia de TIC

\begin{tabular}{|c|c|}
\hline $\begin{array}{l}\text { Avances simultáneos en los ámbitos de internet de las } \\
\text { cosas-IoT }\end{array}$ & $\begin{array}{c}\text { Se deben mitigar los riesgos de la seguridad de la } \\
\text { información. }\end{array}$ \\
\hline El IoT ampliará en gran medida la huella digital & $\begin{array}{l}\text { Se conectarán no sólo personas, organizaciones y recursos de } \\
\text { información, sino también objetos dotados de capacidad de } \\
\text { detención, procesamiento y comunicación en forma digital }\end{array}$ \\
\hline $\begin{array}{l}\text { La nube y otras arquitecturas reducirán las barreras que } \\
\text { impiden el acceso a recursos informáticos escalables }\end{array}$ & $\begin{array}{l}\text { Los servicios en la nube reducen costos fijos de infraestruc- } \\
\text { tura de TIC }\end{array}$ \\
\hline $\begin{array}{l}\text { La inteligencia artificial ayudará a las personas a tomar } \\
\text { mejores decisiones }\end{array}$ & $\begin{array}{l}\text { Los algoritmos deben adaptarse a los datos existentes y a los } \\
\text { objetos fijados }\end{array}$ \\
\hline $\begin{array}{l}\text { Las TIC avanzadas necesitan infraestructuras competi- } \\
\text { tivas y servicios adecuados }\end{array}$ & $\begin{array}{l}\text { Las redes tendrán que soportar los diversos niveles de calidad } \\
\text { del servicio exigidos por usuarios y aplicaciones }\end{array}$ \\
\hline
\end{tabular}

Fuente: International Telecommunications Union (2017).

Las tendencias se pueden tomar como retos que incluyen no sólo planes para ampliar la infraestructura, sino para desarrollar las competencias digitales en la población y de esta manera favorecer la motivación y las habilidades que faciliten su uso tanto en la vida cotidiana como en el campo académico y en el sector productivo (Peña, Cuartas y Tarazona, 2018).

\section{ConcePtualización SObRe COMPETEnCias digitales}

Con el ánimo de precisar la comprensión de las $\mathrm{CD}$, se retoman algunos conceptos. La movilización se refiere a aquellas habilidades y destrezas que permiten buscar, seleccionar críticamente, obtener y procesar información relevante haciendo uso de las TIC para transformarla en conocimiento al mismo tiempo que se es capaz de comunicar dicha información a través de la utilización de diferentes soportes tecnológicos y digitales, además de 
actuar con responsabilidad, respetar las normas socialmente establecidas y aprovechar estas herramientas para informarse, aprender, resolver problemas y comunicarse en distintos escenarios de interacción (Flores y Roig, 2016).

Del concepto anterior se desprende una comprensión de las CD que pretende superar cualquier visión reduccionista sobre ellas. Se destaca, en este caso, la idea de movilización que ayuda a entender que la competencia "no constituye una forma de algoritmo memorizado y practicado repetidamente en vista a asegurar la perennidad y la reproducción" (Tardif, 2008: 8), algo así como un saber mecánico. Esta afirmación implica no aceptar las competencias simplemente como habilidades de tipo instrumental, lo cual se entiende como establecer las relaciones medio-fin, pero sin la reflexión sobre su finalidad. Las competencias digitales son ante todo un saber actuar para resolver situaciones problémicas en diversos contextos.

Asimismo, el desarrollo de las CD contribuye a usar la información para transformarla en conocimiento, la cual al ser abundante invade la vida cotidiana mediante diversas tecnologías, en especial, con el desarrollo de la internet y la web 2.0. No obstante, "la información es en potencia una mercancía que se compra y vende en un mercado y cuya economía se basa en la rareza" (UNESCO, 2005: 19); está relacionada especialmente con el auge tecnológico. Mientras, el conocimiento, aunque tiene en la información un instrumento, posee la finalidad solucionar problemas, producir nuevos conocimientos e innovar para tener un uso social. En ese orden de ideas, la riqueza de la información se encuentra en transformarla en conocimiento.

En este orden de ideas, las CD van más allá de las posibilidades que ofrece el desarrollo tecnológico al considerar inexcusable su comprensión sin estar relacionada con el ámbito de la comunicación, donde se amplía el universo lingüístico de los sujetos y con ello los encuentros y desencuentros, que en el campo de la educación y la pedagogía se traduce en fortalecer el aprendizaje colaborativo y el desarrollo de la pedagogía conectivista (Siemens, 2004). Ahora bien, el desarrollo tecnológico es tan vertiginoso que desborda cualquier vaticinio y, por obvias razones, la educación se convierte en receptora y fuente de creatividad a partir de las distintas innovaciones electrónicas (Bohórquez Ramírez y Silva Monsalve, 2020). Por ejemplo, una característica de la sociedad actual consiste en señalar que es difícil pensar el aprendizaje por fuera de la conexión a redes y, de nuevo, aquí es conveniente el desarrollo de las competencias digitales.

Por último, la competencia digital es aquella que implica el uso creativo, crítico y seguro de las tecnologías de la información y la comunicación para alcanzar los objetivos relacionados con el trabajo, la empleabilidad, el aprendizaje, el uso del tiempo libre, la inclusión y participación en la sociedad (Silva Monsalve y Sandoval Serrano, 2019). Esta competencia supone, además de la adecuación a los cambios que introducen las nuevas tecnologías en la alfabetización, la lectura y la escritura, un conjunto nuevo de conocimientos, habilidades y actitudes necesarias hoy en día para ser competente en un entorno digital (Ministerio de Educación, Cultura y Deporte, 2014).

Se parte del concepto de la incorporación del uso crítico de la tecnología en diversas áreas que resalta la importancia de las competencias digitales para la inserción en el mundo laboral en tiempos en los que parece anunciarse el "fin del empleo" debido al auge de "nuevas tecnologías exponenciales como la robótica avanzada, el transporte autónomo, la inteligencia artificial (AI), los sensores de recogida de datos, la internet de los objetos (IO), la manufactura por impresión 3D, la nanotecnología o la computación cuántica (Coleman, 2017). Recapitulando este apartado, es necesario mencionar que las competencias digitales antes "opcionales", se han convertido en “esenciales" (UNESCO, 2018).

\section{Metodología de investigación}

En el aspecto metodológico se utilizó una investigación mixta. Particularmente, en contextos educativos, los diseños mixtos enriquecen y complementan el análisis de los resultados obtenidos. Sin embargo, no se limitó a la simple recogida de datos de diferente naturaleza, sino que implica combinar la lógica inductiva con la deductiva (Burke, Onwuegbuzie y Turner, 2007), lo cual permite utilizar de forma conjunta técnicas e instrumentos de recopilación de datos integrando los tipos de investigación cuantitativa y cualitativa. Para este artículo el diseño 
utilizado fue mixto secuencial (Tashakkori y Teddlie, 2010). En un inicio, se aplicó un instrumento de autopercepción de $\mathrm{CD}$ a estudiantes, quienes son analizados cuantitativamente desde técnicas estadísticas; más adelante se realizó la triangulación de la información desde teorías y conceptos sobre las competencias digitales.

\section{1. Muestra y recogida de datos}

La población considerada se conformó por alumnos de primeros semestres de las universidades caso de estudio. Se definió una muestra no probabilística de tipo casual (Hernández, Fernández y Baptista, 2014). La muestra se conformó por 69 estudiantes de las universidades USTA y EAN para un total de 138 estudiantes. En la muestra seleccionada se particulariza la distribución de la institución educativa de donde provienen. Al respecto, se evidencia para los estudiantes de la EAN un mayor número en instituciones de carácter privado con un porcentaje de $39.70 \%$ frente a un $31.9 \%$ de la USTA (figura 1 ).

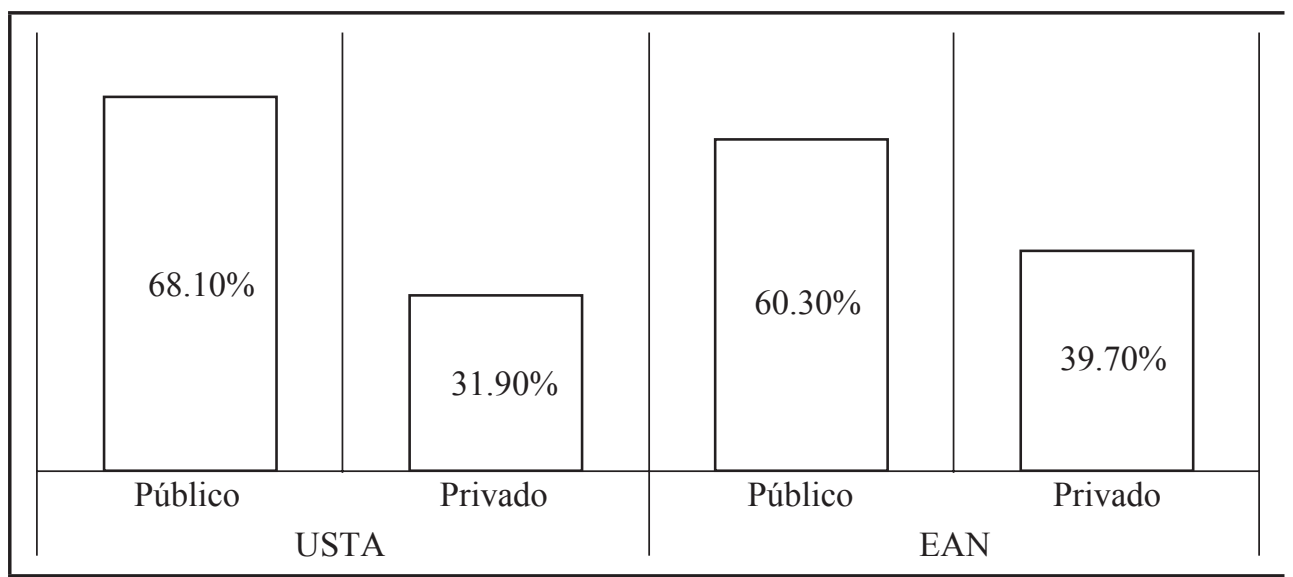

FIGURA 1

Distribución de la muestra de estudiantes por tipo de institución educativa Fuente: elaboración propia.

\section{2. Instrumentos}

Al momento de indagar sobre instrumentos para validar las CD se encontraron diferentes propuestas. Se identificó la investigación sobre el "Diseño y validación de un instrumento de evaluación de la competencia digital del estudiante universitario” elaborada por Juan Jesús Gutiérrez Castillo, Julio Cabero Almenara y Ligia Isabel Estrada Vidal, que es pertinente frente a los objetivos de este artículo. En este sentido, el instrumento propone seis dimensiones distribuidas en a) Dimensión 1. Alfabetización tecnológica (funcionamiento y conceptos de las TIC), b) Dimensión 2. Búsqueda y tratamiento de la información (investigación y manejo de la información), c) Dimensión 3. Pensamiento crítico, solución de problemas y toma de decisiones, $d$ ) Dimensión 4. Comunicación y colaboración, e) Dimensión 5. Ciudadanía digital, f) Dimensión 6. Creatividad e innovación (Gutiérrez Castillo, Cabero Almenara y Estrada-Vidal, 2017). En lo referente al empleo del instrumento se solicitó la autorización de uso a los autores y se llevó a cabo la adaptación para el contexto del artículo en un nivel conceptual y poblacional.

\section{3. Procedimiento}

Se utilizaron técnicas e instrumentos desde la investigación mixta orientados al desarrollo del proyecto. Por consiguiente, los expertos se encargaron de revisar el instrumento, quienes refirieron ajustes en expresiones y TIC no incluidas en las preguntas de la encuesta. Por otra parte, se aplicó la prueba piloto para la adaptación del 
instrumento a 108 estudiantes pertenecientes a diferentes programas, por lo que adaptarlo permitió su contextualización. Posteriormente, su aplicación se llevó de manera formal en los 138 estudiantes pertenecientes a las dos universidades; cabe resaltar la solicitud del consentimiento informado a los participantes.

De igual forma, para el estudio de los datos se trabajó con el análisis de correspondencias múltiples (ACM) y la triangulación de teorías sobre los resultados obtenidos. Para detallar sobre el ACM, se describe como una metodología de estadística multivariada para resumir tablas de contingencia con el fin de abordar los siguientes objetivos: a) Comparar los individuos, generalmente anónimos, para detectar patrones que emergen de los datos. b) Comparar las categorías de las variables y detectar grupos de ellas. c) Explorar relaciones entre las variables a través de sus categorías. d) Describir correspondencia entre individuos y variables. $e$ ) Cuantificar las variables categóricas y reducir de dimensión (Sanabria y Nuñez, 2011). Para resumir, "el ACM permite estudiar un conjunto de individuos descritos por un grupo de variables cualitativas” (Modroño, Fernández y Landaluce, 2004: 207).

El ACM se seleccionó como técnica de análisis para el trabajo al establecer una relación entre las preguntas del instrumento sobre CD. Sin embargo, se complementó con un análisis descriptivo y estableció las semejanzas y diferencias para cada una de las dimensiones en las universidades caso de estudio. Asimismo, desde un enfoque cualitativo se efectúo la triangulación de los datos frente a teorías y algunas percepciones de expertos que se presentan como categorías en las conclusiones.

\section{Análisis y Resultados}

El instrumento para la validación de la CD del estudiante universitario se sustenta en las dimensiones expuestas por $\mathrm{Ng}$ (2012: 1067), donde se definen "dimensión técnica, dimensión cognitiva y dimensión socioemocional para la alfabetización digital". A su vez, el instrumento enfatiza en otras dimensiones, las cuales son consideras indicadores fundamentales para la evaluación en estudiantes y docentes (figura 2).

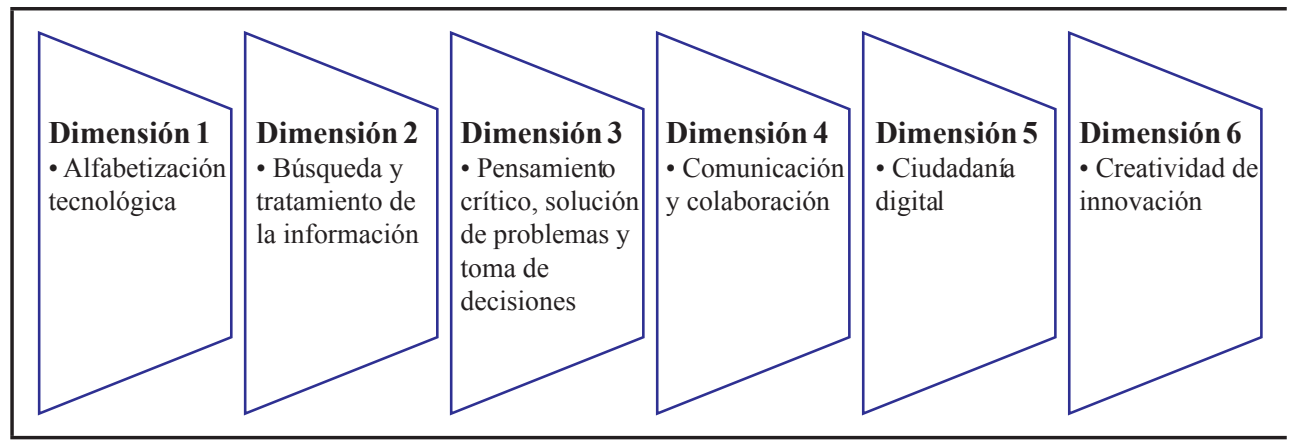

FIGURA 2

Dimensiones para evaluar las CD según Gutiérrez Castillo, Cabero Almenara y Estrada-Vidal Fuente: elaboración propia.

Igualmente, cada dimensión se encuentra conformada por 22 indicadores y 43 preguntas del cuestionario. Ahora bien, la valoración de cada pregunta se encuentra distribuida en una escala nominal del 1 al 10, donde 1 hace referencia a la ineficacia y 10 al dominio en el manejo de la herramienta. De esta manera, se pueden evidenciar los hallazgos de las preguntas, los cuales están representados en un mapa factorial mediante ACM: para la USTA se representan en la gráfica 1 y para la EAN en la gráfica 2.

En la gráfica 1 es evidente cómo se muestran variables significativas $p \leq 0.05$ correspondientes a las preguntas P9, P18, P25, P31, P32, que son resultados correspondientes a los estudiantes de la USTA y que se verán detallados en el análisis descriptivo presentado para cada una de las dimensiones. Por otra parte, las preguntas restantes se encuentran en una escala de valoración entre 0.5 y 1.0, lo cual refleja un índice aceptable de correlación entre cada una de las preguntas. Es importante destacar de los resultados que ninguno se encuentra en un valor inferior a 0. 


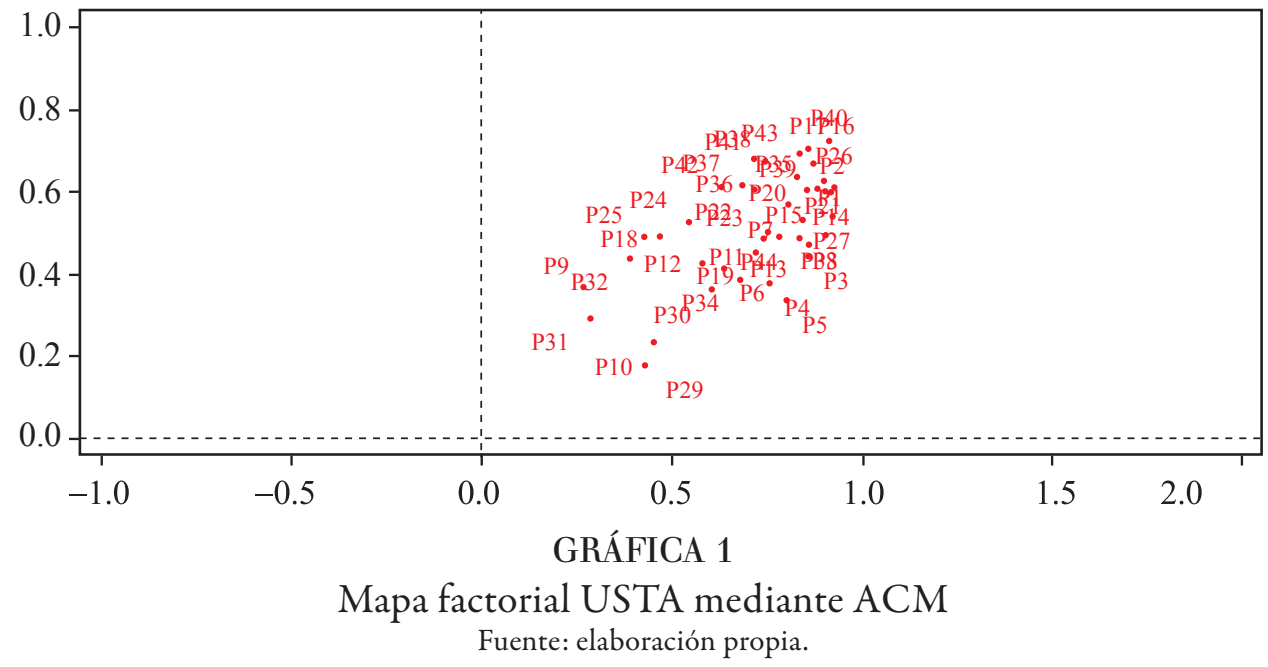

De igual forma, en la gráfica 2 los resultados para la EAN representan una correlación favorable según el mapa factorial para la mayoría de las preguntas. Se evidencian preguntas en una escala entre $>0.2$ y $<0.5$ preguntas: $\mathrm{P} 9$, P10, P30, P31, P41. Las ampliaciones sobre el resultado de estas preguntas se evidencian en el análisis descriptivo de las dimensiones.

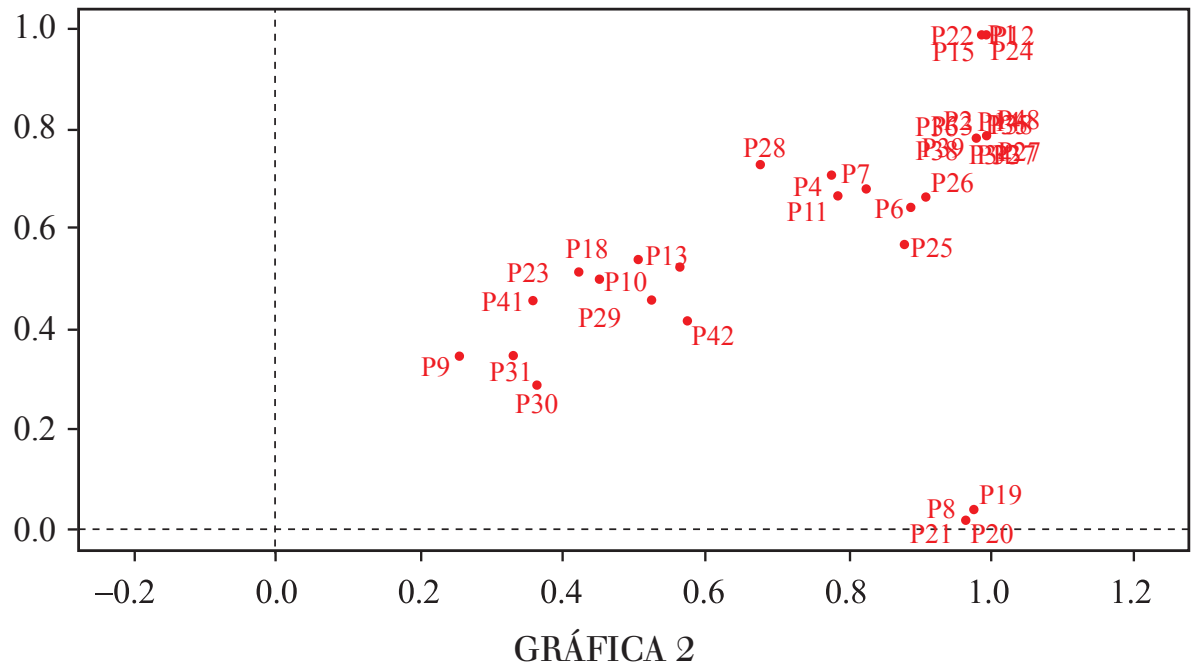

Mapa factorial EAN mediante ACM

Fuente: elaboración propia.

En seguida se presenta el análisis descriptivo para cada una de las dimensiones, donde se resaltan aspectos considerados de especial atención. Se comienza con la dimensión de alfabetización tecnológica, de la cual se presentan los resultados en la tabla 2 y evidencia un alto porcentaje sobre el grado de dominio de las herramientas básicas que permiten el acercamiento a las plataformas, inclusive manejo de los sistemas operativos tanto en dispositivos de escritorio y móviles como en la utilización de navegadores para el acceso a la internet. Para la USTA se mencionan porcentajes por debajo de $70 \%$ respecto al diseño de páginas web y el uso de software para trabajo colaborativo; para la EAN, un porcentaje equivalente a $70.9 \%$ respecto al diseño de páginas web.

Se presentan los resultados para la dimensión 2 sobre búsqueda y tratamiento de la información, donde se evidencia la habilidad para su selección, así como la gestión y manejo utilizando diferentes herramientas. Se menciona para la USTA un porcentaje de $75.2 \%$ en el uso de organizadores gráficos y software para elaborar mapas conceptuales y mentales (tabla 3 ). 
TABLA 2

Resultados para la dimensión 1: alfabetización tecnológica

\begin{tabular}{|c|c|c|}
\hline & $\%$ & $\%$ \\
\hline Indicadores & USTA & EAN \\
\hline $\begin{array}{l}\text { P.1. Soy capaz de utilizar distintos tipos de sistemas operativos instalados en un computador } \\
\text { (Microsoft Windows, Linux, Mac...) }\end{array}$ & 89.5 & 92.6 \\
\hline P.2. Soy capaz de utilizar distintos tipos de sistemas operativos instalados en dispositivos móviles & 82.6 & 94.1 \\
\hline P.3. Soy capaz de utilizar distintos dispositivos móviles (smartphone, tablet, PDAs) & 88.3 & 88.4 \\
\hline $\begin{array}{l}\text { P.4. Navego por la internet utilizando diferentes opciones (Internet Explorer, Mozilla Firefox, } \\
\text { Safari, Opera) }\end{array}$ & 91.2 & 89.8 \\
\hline $\begin{array}{l}\text { P.5. Domino distintas herramientas ofimáticas para el tratamiento de la información tales como } \\
\text { los procesadores de texto, hojas de cálculo, bases de datos... }\end{array}$ & 89.8 & 94.1 \\
\hline $\begin{array}{l}\text { P.6. Investigo y resuelvo problemas en los sistemas y aplicaciones (configurar correo electrónico, } \\
\text { configurar antivirus, desfragmentar el disco duro...) }\end{array}$ & 75.2 & 89.7 \\
\hline P.7. Soy capaz de utilizar distintas herramientas de tratamiento de imagen, audio o video digital & 88.3 & 89.7 \\
\hline $\begin{array}{l}\text { P.8. Me puedo comunicar con otras personas utilizando herramientas de comunicación sincrónica } \\
\text { vía web (chat, servicios de mensajería instantánea, Skype...). }\end{array}$ & 92.6 & 94.2 \\
\hline $\begin{array}{l}\text { P.9. Sé diseñar páginas web utilizando algún programa informático, incluyendo textos, imágenes, } \\
\text { audio, links... }\end{array}$ & 67.9 & 70.9 \\
\hline $\begin{array}{l}\text { P.10. Sé usar software de trabajo colaborativo utilizando las herramientas online tipo Groupware } \\
\text { (Google Apps, BSCW, OpenGroupWare...) }\end{array}$ & 63.3 & 86.9 \\
\hline $\begin{array}{l}\text { P.11. Domino las herramientas de la web } 2.0 \text { para compartir y publicar recursos en línea (blog, } \\
\text { SlideShare, YouTube, podcast...) }\end{array}$ & 79.6 & 92.5 \\
\hline $\begin{array}{l}\text { P.12. Uso de manera eficaz el campus virtual utilizado en mi Universidad (Moodle, Blackboard, } \\
\text { WebCT...) como apoyo al proceso de formación }\end{array}$ & 79.6 & 92.6 \\
\hline $\begin{array}{l}\text { P.13. Me siento competente para utilizar la gestión virtual (procesos académicos administrativos, } \\
\text { servicios de la biblioteca...) de mi Universidad }\end{array}$ & 81.1 & 86.7 \\
\hline
\end{tabular}

Fuente: elaboración propia.

TABLA 3

Resultados para la dimensión 2: búsqueda y tratamiento de la información

\begin{tabular}{|lcc|}
\hline \multicolumn{1}{|c}{ Indicadores } & $\begin{array}{c}\% \\
\text { USTA }\end{array}$ & EAN \\
\hline $\begin{array}{l}\text { P.14. Soy capaz de localizar información a través de diferentes fuentes y bases de datos } \\
\text { disponibles en la red }\end{array}$ & 88.4 & 92.6 \\
$\begin{array}{l}\text { P.15. Sé identificar la información relevante evaluando distintas fuentes y su procedencia } \\
\text { P.16. Soy capaz de organizar, analizar y usar éticamente la información a partir de una } \\
\text { variedad de fuentes y medios }\end{array}$ & 89.7 & 91.2 \\
$\begin{array}{l}\text { P.17. Sintetizo la información seleccionada adecuadamente para la construcción y asimi- } \\
\text { lación del nuevo contenido, mediante tablas, gráficos o esquemas }\end{array}$ & 86.9 \\
$\begin{array}{l}\text { P.18. Uso organizadores gráficos y software en la elaboración de mapas conceptuales y } \\
\text { mentales (CmapTools, Mindomo...), diagramas o esquemas para presentar las relaciones } \\
\text { entre ideas y conceptos }\end{array}$ & 95.2 \\
P.19. Planifico búsquedas de información para la resolución de problemas & 92.6 \\
\hline
\end{tabular}

Fuente: elaboración propia. 
Los resultados de la dimensión 3, reflejan el buen uso sobre el manejo de los recursos y las herramientas digitales para la exploración de temas del mundo actual y la solución de problemas reales. Para la USTA, los estudiantes no poseen un manejo sobresaliente sobre la configuración y resolución de hardware, software y sistemas de redes. Los resultados se ubican en un 75.2 por ciento.

TABLA 4

Resultados para la dimensión 3: identificación de problemas usando las TIC pensamiento crítico y solución del problema

\begin{tabular}{|lcc|}
\hline \multicolumn{1}{|c}{ Indicadores } & $\begin{array}{c}\% \\
\% \\
\text { USTA }\end{array}$ & EAN \\
\hline $\begin{array}{lc}\text { P.20. Soy capaz de identificar y definir problemas o preguntas de investigación utilizando las TIC } \\
\text { P.21. Utilizo los recursos y herramientas digitales para la exploración de temas del mundo actual y la }\end{array}$ & 83.9 & 89.8 \\
solución de problemas reales atendiendo a necesidades personales, sociales, profesionales... & 93.8 \\
$\begin{array}{lc}\text { P.22. Sé analizar los alcances y limitaciones de los recursos TIC } \\
\begin{array}{l}\text { P.23. Configuro y resuelvo problemas que se presenten relacionados con hardware, software y sistemas } \\
\text { de redes para optimizar su uso para el aprendizaje y la productividad }\end{array}\end{array}$ & 89.7 & 92.5 \\
\hline
\end{tabular}

Fuente: elaboración propia.

En cuanto a la dimensión 4, sobre las habilidades de comunicación y colaboración de los estudiantes en redes sociales y académicas, se presentan indicadores favorables para la comprensión cultural y una conciencia global sobre la comunicación en redes. Sin embargo, los estudiantes de las dos universidades adolecen del manejo de habilidades para el uso de redes académicas, al igual que los marcadores sociales para localizar, almacenar y etiquetar recursos de internet. Por otra parte, los estudiantes de la USTA no acceden a Facebook para realizar trabajos colaborativos. Los anteriores resultados se evidencian en la tabla 5.

TABLA 5

Resultados para la dimensión 4: comunicación y colaboración

\begin{tabular}{|c|c|c|}
\hline Indicadores & $\begin{array}{l}\% \\
\text { USTA }\end{array}$ & $\begin{array}{c}\% \\
\text { EAN }\end{array}$ \\
\hline $\begin{array}{l}\text { P.24. Comparto información de interés con mis compañeros empleando una variedad de entornos y } \\
\text { medios digitales }\end{array}$ & 75.2 & 89.8 \\
\hline $\begin{array}{l}\text { P.25. Comunico efectivamente información e ideas a múltiples audiencias usando variedad de medios } \\
\text { y formatos }\end{array}$ & 70.9 & 88.3 \\
\hline $\begin{array}{l}\text { P.26. Soy capaz de desarrollar una comprensión cultural y una conciencia global mediante la comu- } \\
\text { nicación con otros estudiantes y profesionales de otras culturas }\end{array}$ & 82.6 & 91.2 \\
\hline $\begin{array}{l}\text { P.27. Sé utilizar programas informáticos (SlideShare, Google Docs...) y herramientas tecnológicas } \\
\text { para administrar y comunicar información con mis compañeros y otros usuarios en la red }\end{array}$ & $\mathrm{S}$ & 92.6 \\
\hline $\begin{array}{l}\text { P.28. Interactúo con otros compañeros y usuarios empleando las redes sociales (Facebook, Ning, } \\
\text { Twitter) y canales de comunicación (blog, canal YouTube...) basados en TIC }\end{array}$ & 71.2 & 85.1 \\
\hline $\begin{array}{l}\text { P.29. Soy capaz de desenvolverme en redes de ámbito profesional (LinkedIn, ResearchGate, Acade- } \\
\text { mia...) }\end{array}$ & 70.9 & 78.1 \\
\hline P.30. Soy capaz de diseñar, crear o modificar una wiki (Wikispaces, Nirewiki...) & 59.3 & 59.2 \\
\hline P.31. Sé utilizar los marcadores sociales para localizar, almacenar y etiquetar recursos d & 70.9 & 78.1 \\
\hline
\end{tabular}

Fuente: elaboración propia. 
La dimensión 5 sobre ciudadanía digital abarca aspectos como el compromiso ético en el uso de la información digital y de las TIC, inclusive el respeto por los derechos de autor, práctica y uso seguro, legal y responsable de la información y de las TIC. Sobre los resultados presentados en la tabla 6 , se expresan satisfactoriamente el conocimiento y el ejercicio de estas prácticas por parte de los estudiantes. Lo anterior es un aspecto fundamental, pues se entiende que el manejo no ético en la internet ha generado problemáticas importantes para los estudiantes.

TABLA 6

Resultados para la dimensión 5: ciudadano digital

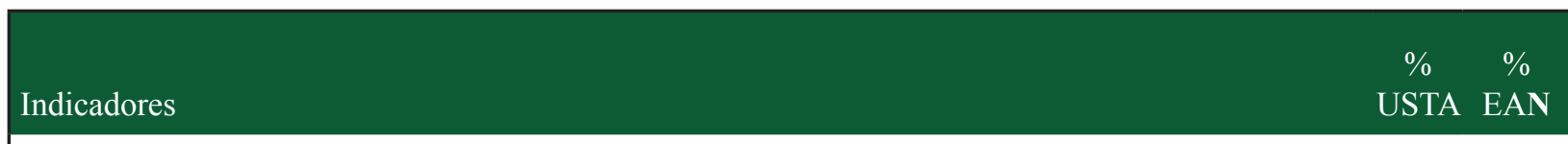

P.32. Asumo un compromiso ético en el uso de la información digital y de las TIC, inclusive el respeto por los derechos de autor, la propiedad intelectual y la referencia adecuada de las fuentes

$92.7 \quad 94.1$

P.33. Promuevo y practico el uso seguro, legal y responsable de la información y de las TIC

$89.8 \quad 92.6$

P.34. Demuestro la responsabilidad personal para hacer realidad el principio "el aprendizaje debe ser a lo largo de la vida" utilizando las TIC

$86.8 \quad 94.1$

P.35. Me considero competente para hacer críticas constructivas juzgando y haciendo aportaciones a los trabajos TIC desarrollados por mis compañeros

P.36. Ejerzo liderazgo para la ciudadanía digital dentro de mi grupo

$86.9 \quad 91.2$

P.37 Exhibo una actitud positiva frente al uso de las TIC para apoyar la colaboración, el aprendizaje y la productividad

$88.3 \quad 92.7$

P.38. Tengo la capacidad de concebir ideas originales, novedosas y útiles utilizando las TIC

$85.3 \quad 91.2$

Fuente: elaboración propia.

De igual manera, para la dimensión sobre creatividad e innovación destacan en los estudiantes resultados favorables en cuanto al manejo de herramientas que permiten explorar la creatividad e innovación en el uso de las TIC. Los resultados están presentes en la tabla 7.

TABLA 7

Resultados para la dimensión 6: creatividad e innovación

\begin{tabular}{|lcc|}
\hline Indicadores & USTA & EAN \\
\hline P.39. Soy capaz de crear trabajos originales utilizando los recursos TIC tradicionales y emergentes & 86.9 & 94.1 \\
\hline $\begin{array}{l}\text { P.40. Identifico tendencias previendo las posibilidades de utilización que me prestan las TIC } \\
\text { P.41. Uso modelos y simulaciones para explorar sistemas y temas complejos utilizando las TIC }\end{array}$ & 81 & 88.4 \\
$\begin{array}{l}\text { P.42. Desarrollo materiales donde utilizo las TIC de manera creativa apoyando la construcción de } \\
\text { mi conocimiento }\end{array}$ & 81.1 & 85.5 \\
\hline \begin{tabular}{l} 
P.43. Soy capaz de adaptarme a nuevas situaciones y entornos tecnológicos \\
\hline
\end{tabular} & 92.7 & 94.2 \\
\hline
\end{tabular}

Por otra parte, al aplicar el instrumento de autopercepción sobre CD al grupo de docentes seleccionados, en la tabla 8 se encuentra el promedio de las respuestas que cuenta con una escala de 1 a 10. Para cada dimensión se tuvo en cuenta el indicador de pregunta más bajo. 
TABLA 8

Resultados para la dimensión 6: creatividad e innovación

\begin{tabular}{|c|c|c|}
\hline Dimensión & Indicador & Promedio \\
\hline & $\begin{array}{l}\text { Sé usar software de trabajo colaborativo utilizando las herramientas } \\
\text { online tipo Groupware (Google Apps, BSCW, OpenGroupware...) }\end{array}$ & 6 \\
\hline D1. Alfabetización tecnológica & $\begin{array}{l}\text { Uso de manera eficaz el campus virtual utilizado en mi Universidad } \\
\text { (Moodle, Blackboard, WebCT, MOOC) como apoyo al proceso de } \\
\text { formación }\end{array}$ & 6.8 \\
\hline $\begin{array}{l}\text { D2. Búsqueda y tratamiento de } \\
\text { la información }\end{array}$ & $\begin{array}{l}\text { Uso organizadores gráficos y software para la realización de mapas con- } \\
\text { ceptuales y mentales (CmapTools, Mindomo...), diagramas o esquemas } \\
\text { para presentar las relaciones entre ideas y conceptos }\end{array}$ & 7.7 \\
\hline $\begin{array}{l}\text { D3. Identificación de problemas } \\
\text { usando las TIC pensamiento } \\
\text { crítico y solución del problema }\end{array}$ & $\begin{array}{l}\text { Configuro y resuelvo problemas que se presenten relacionados con } \\
\text { hardware, software y sistemas de redes para optimizar su uso para el } \\
\text { aprendizaje y la productividad }\end{array}$ & 6.4 \\
\hline $\begin{array}{l}\text { D.4. Comunicación y colabo- } \\
\text { ración }\end{array}$ & $\begin{array}{l}\text { Soy capaz de diseñar, crear o modificar una wiki (Wikispaces, Ni- } \\
\text { rewiki...) }\end{array}$ & 6.7 \\
\hline D.5. Ciudadano digital & $\begin{array}{l}\text { Sé utilizar los marcadores sociales para localizar, almacenar y etiquetar } \\
\text { recursos de la internet }\end{array}$ & 6.9 \\
\hline D.6. Creatividad e innovación & $\begin{array}{l}\text { Uso modelos y simulaciones para explorar sistemas y temas complejos } \\
\text { utilizando las TIC }\end{array}$ & 7.5 \\
\hline
\end{tabular}

En la mayoría de los casos se evidencia cómo coinciden los resultados obtenidos tanto para docentes como para estudiantes. Sin embargo, para la dimensión sobre ciudadanía digital, los docentes sí reflejan un dominio sobre el manejo de redes académicas a diferencia de los estudiantes.

\section{Conclusiones}

Los resultados del estudio se presentan mediante una aproximación a los referentes teóricos de las CD permitiendo identificar las fortalezas y aspectos por mejorar de los estudiantes de las dos universidades caso de estudio. Retomando el modelo de alfabetización digital de $\mathrm{Ng}$ (2012), donde se proponen tres dimensiones: a) cognitiva, b) técnica y c) socioemocional (Ramírez y Quiros Silva, 2021), se enlistan a continuación aspectos fundamentales para la caracterización de los estudiantes en programas de formación en línea.

Desde la dimensión cognitiva sobre el uso de herramientas y la alfabetización informacional, se perciben las capacidades de los estudiantes para acceder al trabajo en los ambientes virtuales de aprendizaje (Silva Monsalve, Mendoza Girado y Girado Ruiz, 2018). Para las dos universidades se evidencia el manejo de sistemas operativos, facilidad de acceso a la internet, entre otros. Los anteriores resultados permiten evidenciar un nivel aceptable de las CD desde la dimensión cognitiva para los estudiantes de primeros semestres; sin embargo, se sugiere una mayor atención en el uso de organizadores gráficos: mapas mentales, mapas conceptuales, por mencionar algunos.

Continuando desde la dimensión de comunicación y colaboración, son requeridas habilidades cognitivas y técnicas. Ante ello, se debe instruir a los estudiantes en el empleo de herramientas de trabajo colaborativo implementadas por medio de las TIC, es decir, incentivar el aprendizaje colaborativo, así como lo han mencionado Avello y Duart (2016) en el sentido que el aprendizaje colaborativo no ha desaparecido con las TIC, más bien se ha fortalecido y adaptado a los nuevos entornos que se han configurado y está presente como elemento esencial para lograr el aprendizaje en los ciudadanos del siglo XXI. Ahora bien, es importante la dimensión que se establece desde el uso crítico de las TIC, pues es evidente que desde una dimensión socioemocional los estudiantes de las dos universidades se encuentran fortalecidos. Al respecto, es esencial resaltar el manejo responsable en el uso de redes. 
Finalmente, es primordial resaltar las categorías resultantes sobre las CD y su relación con su proceso de formación, las cuales deben ser consideradas para la caracterización de los estudiantes y docentes en programas de formación en línea terminado ( $\mathrm{Ng}, 2012)$. Se enumeran a continuación:

a) La dimensión cognitiva: el estudiante debe estar en aptitud de indagar, seleccionar y aplicar con capacidad crítica las TIC de acuerdo con los contextos de su proceso de enseñanza.

b) La dimensión socioemocional: acerca del manejo responsable de las TIC para comunicarse, socializarse y aprender. Debe ser una competencia fundamental en los estudiantes, toda vez que la seguridad en las redes no es un aspecto.

c) Sobre la creatividad: los estudiantes deben adquirir desde las TIC habilidades para proponer soluciones en diferentes situaciones.

d) La comunicación y colaboración: son habilidades que se proponen para el siglo XXI. Así, los estudiantes deben utilizar medios y entornos digitales para comunicarse y trabajar de forma colaborativa, incluso a distancia, para apoyar el aprendizaje individual y contribuir al aprendizaje de otros. Quizás, ésta sea la habilidad que los estudiantes deben desarrollar para la humanización en las TIC.

e) Investigación y manejo de información: la extensa colección de información que se encuentra en la internet debe permitir desarrollar en los estudiantes la capacidad de seleccionar fuentes confiables y apropiadas. Los estudiantes aplican herramientas digitales para obtener, evaluar y usar información.

f) Pensamiento crítico, solución de problemas y toma de decisiones: los estudiantes usan habilidades de pensamiento crítico para planificar y conducir investigaciones, administrar proyectos, resolver problemas y tomar decisiones informadas usando herramientas y recursos digitales apropiados.

g) La ciudadanía digital: los estudiantes comprenden los asuntos humanos, culturales y sociales relacionados con las TIC y practican conductas legales y éticas.

Resulta conveniente cerrar este escrito enunciando la siguiente reflexión: las tecnologías no son solamente un instrumento en el proceso de enseñanza y aprendizaje, sino que se constituyen en una mediación entre el estudiante, el docente y la formación, que van determinando las formas de ser, pensar y actuar (Silva Monsalve y Montañéz Sánchez, 2019). Dado el alcance de este artículo se considera pertinente describir e indagar sobre este tema. Por el momento, basta señalar el reto investigativo al evidenciar que las nuevas generaciones que están por ingresar a la educación superior tienen nuevas formas de conocer, de aprender y, por tanto, de ser. Por lo anterior, se debe propender por la calidad académica (Loría, 2018). Estas situaciones convocan a pensar en la necesidad de asumir con dinamismo e ingenio la comprensión de la experiencia humana en los tiempos de las tecnologías de la información y la comunicación.

\section{REFERENCIAS}

Avello, R. y Duart, J. M. (2016). Nuevas tendencias de aprendizaje colaborativo en e-learning. Claves para su implementación efectiva. Estudios pedagógicos, 42(1) 271-282.

Bohórquez, G. y Silva Monsalve, A. (2020). Incidencia del pensamiento lógico matemático en la formación de estudiantes en programas de ingeniería. Una revisión documental, en E. Serna, Revolución en la Formación y la capacitación para el Siglo XXI (pág. 663). Medellín: Instituto Antioqueño de Investigación.

Bohórquez Ramírez, G., y Quiros Silva, F. S. (2021/07). Análisis de los estilos de aprendizaje en estudiantes 
de formación en línea mediante el test de dominancia cerebral: Un caso de estudio, en E. Serna, Ciencia transdisciplinar para el desarrollo y la supervivencia de la humanidad (pág. 458). Medellín: Instituto Antioqueño de Investigación.

Burke, J., Onwuegbuzie, A., \& Turner, L. (2007). Toward a definition of mixed methods research. Journal of Mixed Methods Research, 1(2), 8-22.

Coleman, G. (2017). The next industry revolution will not be televised. Retrieved from www.weforum.org/agen$\mathrm{da} / 2016 / 01 /$ the-next-industry-revolutionwill-not-be-televised/

Esteve, F. y Gisbert, M. (2013). Competencia digital en la educación superior: instrumentos de evaluación y nuevos entornos. Información, Tecnología y Conocimiento, 10(3), 29-43.

Modroño, J. I., K., Fernández, K., \& Landaluce, M. I. (2004). ACMy Statis dual ponderado. Dos técnicas complementarias para analizar una visión de la cultura de la Universidad. Estadistica Española, 46(156), 205-228.

Figel, J. (2009). Competencias clave para el aprendizaje permanente. Al tablero (52). Disponible en http://www. mineducacion.gov.co/1621/article-210023.html

Flores, C. y Roig, R. (2016). Diseño y validación de una escala de autoevaluación de competencias digitales para estudiantes de pedagogía. Pixel-Bit. Revista De Medios y Educación, 48, $209-224$.

Gutiérrez Castillo, J. R., Cabero Almenara, J. y Estrada Vidal, L. I. (2017). Diseño y validación de un instrumento de evaluación de la competencia digital del estudiante universitario. Espacios, 38(10), 1-27.

Hernández, R., Fernández, C. y Baptista, P. (2014). Metodología de la investigación. México: McGraw Hill.

Informe Horizon. (2016). Resumen Informe Horizon. Educación Superior. Disponible en https://intef.es/ wp-content/uploads/2016/03/Resumen_Horizon_Universidad_2016_INTEF_mayo_2016.pdf

INTEF. (2017). Marco común de competencia digital docente. Madrid: Ministerio de Educación, Cultura y Deporte.

International Telecommunications Union. (2017). Informe sobre la medición de la sociedad de la información de 2017. Ginebra: ITU.

Loría, E. (2018). Productividad y productivismo en las publicaciones académicas. CIENCIA ergo-sum, 25(3).

Martínez Morales, E. y Silva Monsalve, A. (2018). Factores asociados al éxito académico en programas de Educación Virtual y a Distancia: informe de avance de investigación. Universidad Santo Tomás. Disponible en https:// repository.usta.edu.co/handle/11634/13827

Ministerio de Educación Nacional. (2013). Competencias TIC para el desarrollo profesional docente. Disponible en https://www.mineducacion.gov.co/1759/articles-339097_archivo_pdf_competencias_tic.pdf

Ministerio de Educación, Cultura y Deporte. (2014).BOE. Disponible en https://www.boe.es/buscar/pdf/2014/ BOE-A-2014-2222-consolidado.pdf

Muñoz Rojas, H. (2016). Mediaciones tecnológicas: nuevos escenarios de la práctica pedagógica. Praxis \& Saber, 7(13), 199-221.

New Media Consortium-EDUCAUSE. (2018). Horizon Report 2018. Higher Education Edition. EDUCAUSE. Ng, W. (2012). ¿Can we teach digital natives digital literacy? Computers \& Education, 59(3), 10651-1078.

NMC Horizon. (2016). Resumen Informe Horizon. Educación Superior. Austin: INTEF.

Peña, H., Cuartas, K. y Tarazona, G. (2018). La brecha digital en Colombia: un análisis de las políticas gubernamentales para su disminución. Redes de Ingeniería, 8, 59-71.

Pérez, R., Miño, E., Miño, M. y Feijoó, W. (2017). Competencias para la educación superior en el Ecuador del siglo XXI: responsabilidad social y tecnologías. In Crescendo, 8(2), 309-320.

PLANIED. (2016). Competencias de educación digital. Colección de marcos pedagógicos. Argentina: EDUCAR. 
Sanabria, G. y Nuñez, F. (2011). Análisis multivariado de datos: aproximación didáctica. XIII Conferencia Interamericana de Educación Matemática. Brasil: CIAEM.

Siemens, G. (2004). Conectivismo: una teoría de aprendizaje para la era digital. Disponible en http://www.diegoleal.org/docs/2007/Siemens(2004)-Conectivismo.doc

Silva Monsalve, A. M. y Montañéz Sánchez, L. (2019). Aprendizaje psicomotriz en el área de educación física, recreación y deportes mediado por el uso de software educativo. Retos: nuevas tendencias en educación física, deporte y recreación, 36, 302-309.

Silva Monsalve, A. M., Girado, J. y Ruiz, A. I. (2018). Prevención del consumo de sustancias psicoactivas. Un aporte desde la neurociencia y el aprendizaje basado en proyectos ABP. Revista Iberoamericana de Educación, 78(1), 107-126.

Silva Monsalve, A. M. y Sandoval Serrano, M. A. (2019). Organizadores gráficos: estrategia didáctica en ambientes virtuales mediada por la identificación de estilos de aprendizaje. CITAS, 5(1), 89-108.

Tardif, J. (2008). Desarrollo de un programa por competencias: de la intención a su implementación. Profesorado. Revista de currículum y formación del profesorado, 16(3), 36-45.

Tashakkori, A., y Teddlie, C. (2010). SAGE Handbook of mixed methods in social \& behavioral research. SAGE.

UNESCO. (2005). Hacia las sociedades del conocimiento: informe mundial de la UNESCO. Disponible en https:// unesdoc.unesco.org/ark:/48223/pf0000141908

UNESCO. (2018). Las competencias digitales son para el empleo y la inclusión social. UNESCO. Disponible en http://www.unesco.org/new/es/media-services/single-view-tv-release/news/las_competencias_digitales_son_esenciales_para_el_empleo_y_l/

\section{Nota}

[1] El trabajo se deriva la investigación titulada "Determinación de factores de rendimiento asociado al éxito académico en programas de educación virtual y a distancia” (Martínez Morales y Silva Monsalve, 2018).

\section{BY-NC-ND}

